\title{
Biological effects of tandem shock waves demonstrated on magnetic resonance
}

\author{
Benes $\mathrm{J}^{1}$, Zeman $\mathrm{J}^{1}$, Pouckova $\mathrm{P}^{1}$, Zadinova $\mathrm{M}^{1}$, Sunka $\mathrm{P}^{2}$, Lukes $\mathrm{P}^{2}$ \\ Institute of Biophysics and Informatics, First Faculty of Medicine, Charles University in Prague, Prague, \\ Czech Republic. jan.zeman@If1.cuni.cz
}

\begin{abstract}
Objectives: The shock wave is used for the treatment of kidney stones, eventually of gall stones, for more than 20 years. It is a pressure wave, which breaks through soft tissues easily and it is possible to focus it into a small volume. The excellent results of the treatment of concrements led to considerations about another usage of the shock wave. The research is now concentrated on the possibility of the damage to tumour tissues. Methods: In contrast to concrements tumour tissues are not different from healthy tissues as for their acoustic attributes. That is why a new source of shock waves was used in this work. The source allows generating two successive shock waves focused into a common focus, so-called tandem shock waves. The biological effects of the tandem shock waves generated by the new source on rats hepatic tissue and rabbit femoral muscle in vivo were studied in this work. The damage is demonstrated by magnetic resonance imaging.

Results: MR images showed tissue damage in focus. There was damage of the liver tissue, muscle and also stomach wall.

Conclusions: We found that the tandem shock waves are able to damage the acoustically homogeneous soft tissue in the focus, i.e. in the depth. In tissues in front of the focus, there is, however, no damage (Fig. 10, Ref. 15). Full Text in PDF www.elis.sk.

Key words: electrical discharges in water, focused shock waves, cavitations, tandem shock waves.
\end{abstract}

The outcomes achieved in the concrement disintegration $(2$, 6-8) initiated a research of a further use of shock waves in medicine. The attractiveness of shock waves results from the fact that they are the only form of energy penetrating through tissues (without damaging them up to a certain threshold) and are able to concentrate energy into a small volume $(3,4)$. The shock waves are used in orthopaedics for the treatment of insertion syndromes and the main interest is in the field of their use for destruction of cancer tissues (5). In contrast to concrements, which have acoustic impedance different from that of surrounding soft tissues, the cancer tissue is essentially not different from the healthy tissue in acoustic properties. Generators of shock waves for the destruction of concrements were developed with the aim to minimize side effects and thus, they cause nearly no damage to soft non-aerated tissues (9). Thus, a new generator of shock waves was developed at the Institute of Plasma Physics. The new generator is based

${ }^{1}$ Institute of Biophysics and Informatics, First Faculty of Medicine, Charles University in Prague, Prague, Czech Republic, and ${ }^{2}$ Institute of Plasma Physics AS CR, v.v.i. Prague, Czech Republic

Address for correspondence: J. Zeman, Charles University in Prague, First Faculty of Medicine, Institute of Biophysics and Informatics, Salmovska 1, Praha 2, CZ-120 00, Czech Republic.

Phone: +420.224965852 , Fax: +420.224965843

Acknowledgements: This work was supported by the Grant Agency of the Czech Republic under contract No. GA202/09/1151 and grant MSM 0021620808. We acknowledge Vít Herynek and Monika Dezortová (both Institute for Clinical and Experimental Medicine) for performing MRI scans of the animals. on a concept that if a local effect of the shock wave should be achieved in an acoustically homogeneous environment, then either the effect of cavitations should be used or two successive shock waves should be generated (10), where the first one produces an acoustic non-homogeneity and the second one is damped in this non-homogeneity $(1,9,11,12)$. The principle of the shock wave generation by the new source is based on a multichannel development, i.e. if a composite electrode is situated in strongly conductive water, many discharge channels are produced in this medium $(13,14)$. This multichannel generator was used to verify effects on erythrocytes, lymphocytes and cancer cell lines. After an in vivo exposure of solid tumours, the destruction was histologically demonstrated. In this approach, it is necessary to document the damage in the depth at a particular site, provided that surface structures of tissues, which are shock waves passing through, are not damaged. Given the fact that cancer tissues and healthy soft non-aerated tissues are not considerably different one from another in terms of their acoustic characteristics, we employed imaging by magnetic resonance and exposed the rat liver and rabbit femoral muscle in vivo. The aim of this was to use the imaging technique, and subsequently after dissection the histological examination, for the demonstration of the deep selective damage at a defined site.

\section{Material and methods}

\section{Generator}

The formerly developed shock wave generator was used, in which two cylindrical pressure waves are directed to a common 
focus with the help of a metallic parabolic reflector. The second shock can be generated with different time delays after the first one. The concept of tandem shocks is necessary to achieve the local action on the acoustically homogeneous environment (as e.g. soft tissue). The first shock produces acoustic non-homogeneity and cavitations in the tissue and the second shock is dissipated in them. Collapsing cavitations produce secondary short-wave shocks, which are capable of interaction with cell-sized formations.

The generation of focused tandem shock waves is based on focusing of cylindrical pressure waves produced in a multichannel discharge on a composite anode in highly conductive water. The composite anode consists of cylindrical electrodes of stainless steel (A2 - $\varnothing 60 \times 70 \mathrm{~mm}, \mathrm{~A} 1-\varnothing 77 \times 20 \mathrm{~mm}$ ) coated with a thin layer of porous ceramic material applied on the anode surface by the plasma spraying technology. Because of differences in the conductivity and permittivity between water and the ceramic layer, the intensity of the electric field on the anode surface is many times higher compared with the bare metallic electrode of the same dimensions. In the highly conductive solution, many short (less than $1 \mathrm{~mm}$ in length) discharged channels are produced. They are almost homogeneously distributed on the anode surface. Every expanding discharged channel forms a hemispherical pressure wave and the superimposition of all these waves results in a cylindrical pressure wave propagating from the anode surface. Cylindrical pressure waves are focused by a metallic parabolic reflector (cathode) and transformed to the shock wave in the vicinity of the focus.

\section{Experimental procedures}

Six Wistar strain rat males from the company ANLAB s.r.o. and laboratory rabbit were used in the experiment. Rats body weight was of 280-300 g and they were fed with the ST1 diet. Rabbit body weight was 3100 g. The exposure to shock waves was performed under anaesthesia. Thus, the rats and rabbit were narcotized with intramuscularly administered solution of Narkamon and Xylazin. The solution was prepared as follows: $2 \mathrm{ml}$ of Narkamon per kg of body weight, $0.7 \mathrm{ml}$ of Xylazin and $2.3 \mathrm{ml}$ of water for injection. The rat was depilated in the region above the liver, fixed in a special holder and situated in the experimental device with having its liver in the focus. The rabbit was depilated onthe hind leg and situated in the experimental device with having its femoral muscle in the focus. Tandem shock waves were applied at two-seconds intervals for rats and one and half-second intervals for rabbit for a period of $30 \mathrm{~min}$. This corresponds to 900 shocks for rats and 1800 shocks for rabbit in the focus area. After the exposure to the shock waves, the rats and rabbit were transferred to NMR scanning.

\section{MRI scanning}

MR images of rats were obtained using a 4.7T Bruker Biospec spectrometer equipped with a commercially available resonator coil (Bruker, Germany). We used a standard T2*-weighted gradient echo sequence, repetition time $\mathrm{TR}=80 \mathrm{~ms}, \mathrm{TE}=3.4 \mathrm{~ms}$, slice thickness $=2 \mathrm{~mm}, \mathrm{FOV}=6 \mathrm{~cm}$, and matrix $256 \times 256$. During MR examinations, the rats were anesthetized by spontaneous breathing of isofluran (Foran, Abbot, Czech Republic). For induction, a 3\% concentration of the anesthetic in air was used, and a $1-2 \%$ concentration was used for maintenance. MR images of rabbit were obtained using a MR tomograph Siemens Magnetom Trio $3 \mathrm{~T}$. We used repetition time $\mathrm{TR}=642 \mathrm{~ms}, \mathrm{TE}=20 \mathrm{~ms}$ and slice thickness $=2 \mathrm{~mm}$. Rabbit was anesthetized by the solution of Narkamon and Xylazin.

The scans were obtained on the first, third and seventh days after the exposure to shock waves. The dissection was performed on the seventh day after the exposure.

\section{Results}

Here, we show MR images of two rats and rabbit.

$\mathrm{n}$ axial scans of the first rat (Figs 1,2 and 3) the arrow indicates the site of the damage produced in the liver region. The damage is situated in the posterior part of the liver and partially also involves the stomach wall. The lesion became clearly visible on the third day together with a huge artefact caused by a blood clot which remained between the lobes.

On the seventh day after the exposure, the rat was dissected. According to MRI scans, we located the damage and obtained its

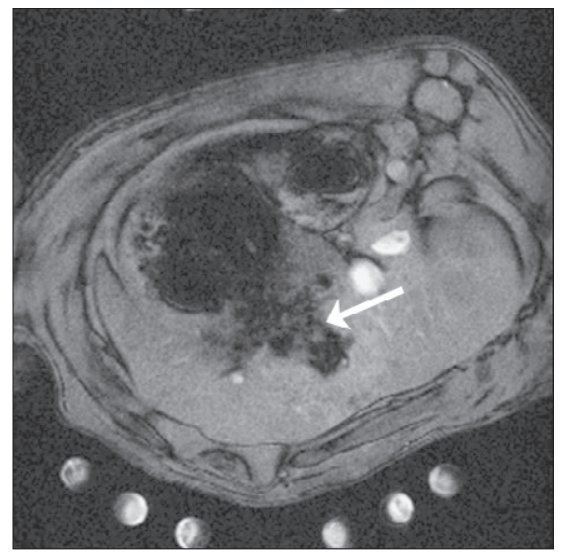

Fig. 1. MRI scan 1 day after the exposure, the first rat.

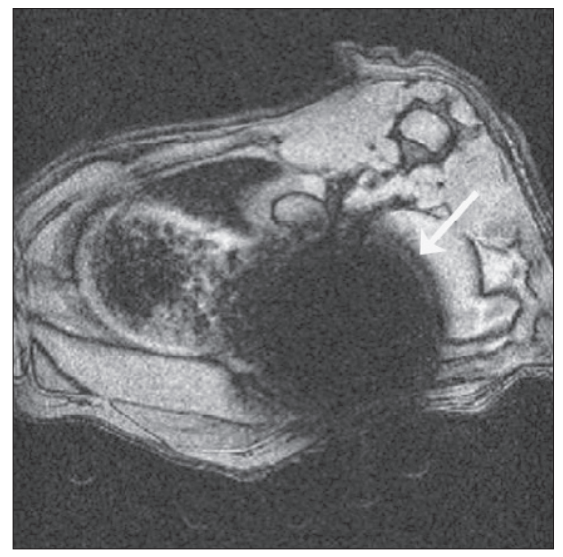

Fig. 2. MRI scan 3 days after the exposure, the first rat.

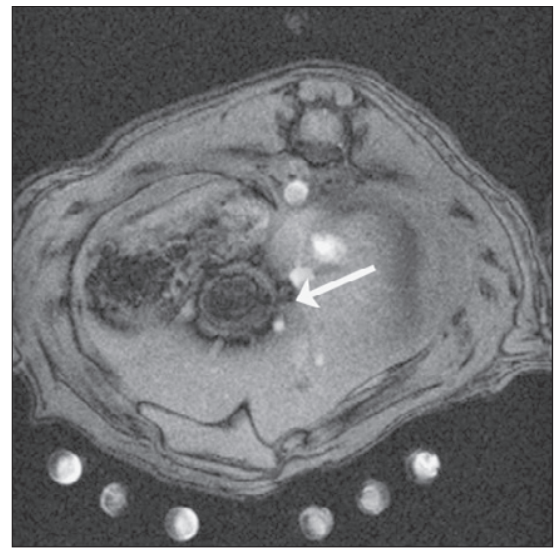

Fig. 3. MRI scan 7 days after the exposure, the first rat. 


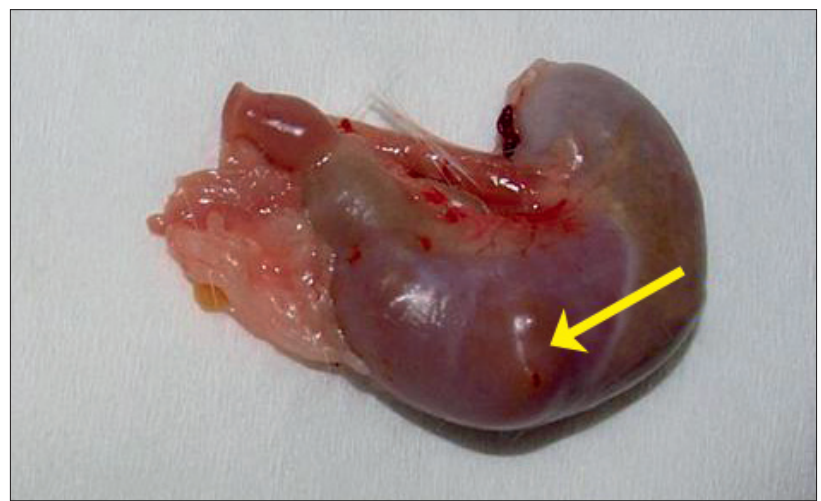

Fig. 4. The stomach of the first rat with detectable thinner wall and vaulted contents.

photo of the posterior wall of the liver with a lesion. In Figure 4 , the adjacent stomach wall is shown, in which the damage can also be seen, the stomach contents being moderately vaulted and the wall thickness being moderately reduced. It is loosened on the inner side. These findings correspond with MR scans. We did not find any other damage.
In Figures 5 and 6 there are MRI scans of the second rat obtained 1 and 3 days after the exposure. A lesion can be seen in the anterior part of the liver. The bounded lesion was detectable during all the MR sessions.

On the seventh day after the exposure, dissection was carried out. In the liver, we found a lesion, the location of which corresponded to MRI scans. This lesion was sharply delimited. We did not find any other damage. The liver with the visible lesion is shown in Figure 7.

Figures 8-10 show MR images of rabbit femoral muscle. Femoral muscle was selected the one leg served as experimental and the other as control. Experimental leg is shown in all pictures on the right side. It can be seen that the soft tissues of the femoral muscle are injured due to the shock wave (arrow). In the focal point haematoma developed and around it an oedema is visible. Control leg is without damage. Imaging was carried out 1 (Fig. 8), 3 (Fig. 9) and 7 (Fig. 10) days after the application of a shock wave.

Scanning and subsequent dissection demonstrated the fact that the shock wave damaged soft tissues. The damage occurred in the region of the focus, and structures above the focus were not damaged. Thus, the shock wave did not damage the soft tissues in front of the focus.

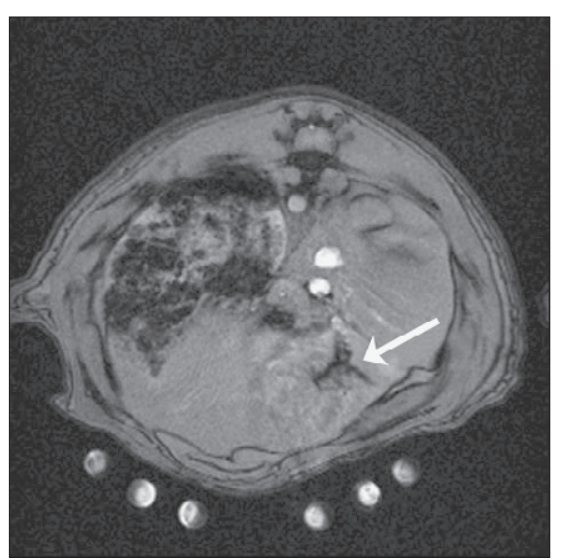

Fig. 5. MRI scan 1 day after the exposure, the second rat.

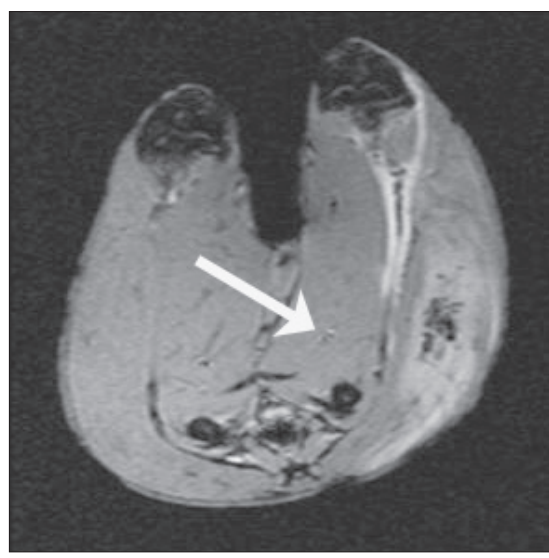

Fig. 8. MRI scan 1 day after the exposure, rabbit.
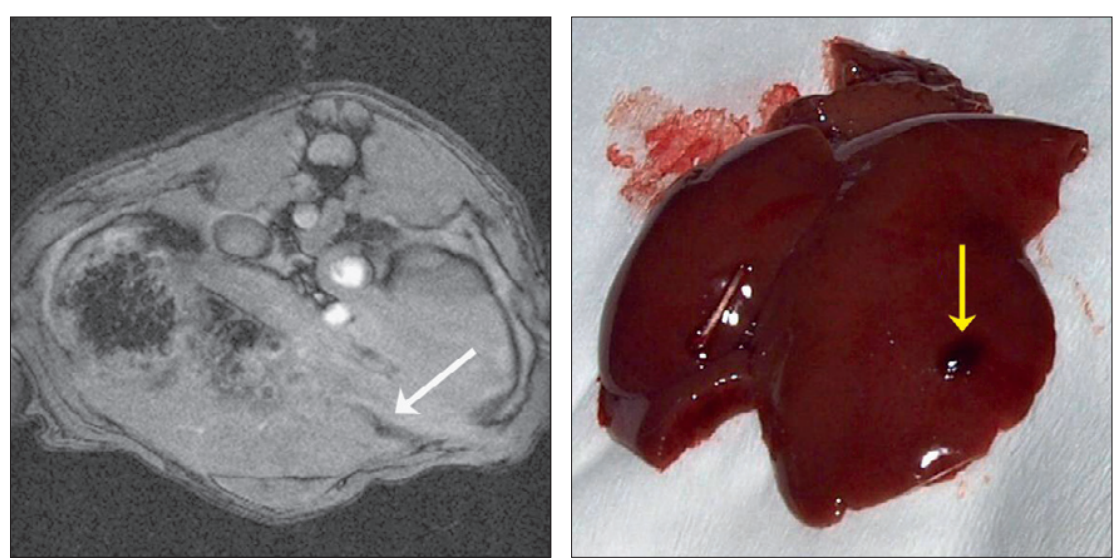
second rat.

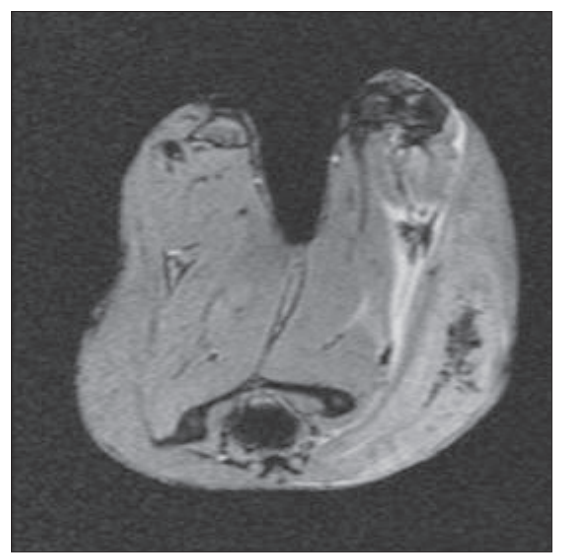

lobe, the second rat.

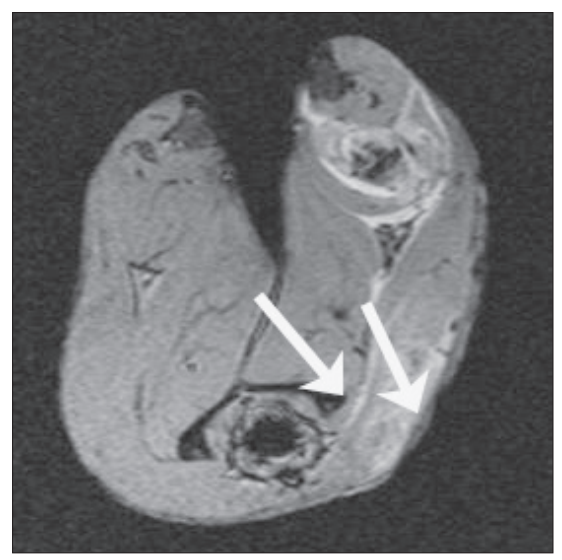

rabbit.
Fig. 10.
rabbit. 


\section{Discussion}

In our experiment, we used a new source of shock waves based on the multichannel discharge in composite anode pores. Due to the fact that the anode is divided into two parts, it is possible to generate tandem shock waves, which are two shock waves subsequently delivered with a short time delay. Thereafter, interaction between the two shock waves occurs in the focus. The second wave comes into the environment with acoustic non-homogeneities produced by the first wave.

The damage occurred in all experimental animals. The experimental results indicate that tandem shock waves generated by the new source are able to damage soft tissues in the focus, i.e. in an acoustically homogeneous environment. MR images show, that it can damage hepatic tissue, stomach wall and femoral muscle. The damage is appropriately delimited and the tissues before the focus remain without any damage. The focal point is deeper under the surface. Shock wave penetrates through the structures before focal point without damage and damage occurs in focal point. The first shock produces acoustic non-homogeneity and cavitations in the tissue and the second shock is dissipated in them. Collapsing cavitations produce secondary short-wave shocks, which are capable of interaction with cell-sized formations. It leads to cell damage in focal point. We can observe it on MR images. During the following days the tissue regenerates. These results support the formerly performed experiment by Beneš et al. (2007).

Magnetic resonance imaging seems to be a suitable tool for lesion monitoring, however, attention should be paid to artifacts caused by possible blood clots, which can migrate into the abdominal cavity and mask the real extent of the lesion.

\section{Conclusion}

The target of the presented experiment was to establish in vivo effects of the new shock wave generator on soft tissues. For this purpose, we used six laboratory rats and one rabbit. The tandem shock waves were applied in such a way that the focus was in the liver area and in femoral muscle.

We found that the tandem shock waves are able to damage the acoustically homogeneous soft tissue in the focus, i.e. in the depth. In tissues in front of the focus, there is, however, no damage. The lesions were detected on MR scans as well as during the dissection. The effects should also be supported by further experiments.

\section{References}

1. Beneš J, Štuka Č, Fořtová H, Chmel J, Šunka P, Klener P. Biological effects of interacting shock waves. A model study of effects of Irv with the use of the determination of the erythrocyte haemolysis. Sbor Lek 1997; 98 (4): 277-282.

2. Chaussy C, Schmiedt E, Jocham D, Brendel W, Forssmann B, Walther V. First clinical experience with extracorporeally induced destruction of kidney stones by shock waves. J Urol 1982; 127: 417-20.
3. Coleman AJ, Saunders JE. A review of the physical properties and biological effects of the high amplitude acoustic fields used in extracorporeal lithotripsy. Ultrasonics 1993; 31 (2): 75-89.

4. Lingeman JE. Contemporary extracorporeal litotripsis. Urol Listy 2004; 2: 5-11.

5. Beneš J. Introduction of a new litotriptor into clinical practice and unusual applications of shock waves. Praha, 2000. 146 pp. Charles University, First Medical Faculty. Habilitation thesis.

6. Beneš J, Šunka P, Kordač V, Jirsa M, Štuka C, Mirejovský P. Preclinical verification of possible extracorporeal lithotripsy of gall stones in experimental animals. Sbor Lek 1987; 89 (5): 129-133.

7. Beneš J, Stuka C, Chmel J, Kordač V, Sunka P, Simon V, Jansta J, Zabka J, Rychlik I, Tluchoř Z. First experience with a Czechoslovak device for the treatment of renal concrements by litotripsis with shock waves. Prakt Lek 1989; 69 (23): 884-887.

8. Beneš J, Šunka P, Kordač V, Bárta Z, Štuka Č, Figura Z, Jirsa M. Equipment for clinical use of extracorporeal lithotrypsis of bile concrements. 261485. ČR. A61B17/22. 1990-01-02.

9. Beneš J, Šunka P, Hani AB. Study of haemolysis after the action of a shock wave produced by a new method with the help of the multichannel discharge. Sbor Lek 2001; 102 (1): 29-35.

10. Loske AM, Prieto FE, Fernandez F, van Cauwelaert J. Tandem shock wave cavitation enhancement for extracorporeal lithotripsy. Physics in medicine and biology. NOV 21 2002; 47 (22): 3945-3957. ISSN 0031-9155.

11. Stelmashuk V, Šunka P. Mutual interaction of two shock waves with a different time delay. Czechoslovak Journal of Physics. 2006, Vol. 56, pp. B396-B400. Available at WWW: <http://www.springerlink.com/content/ ap15j125444350j6/>.

12. McClure S, Dorfmüller C. Extracorporeal Shock Wave Therapy: Theory and Equipment. Clin Techn Equine Pract 2003; 2 (4): 346-357.

13. Šunka P, Babický V. Method of generating espacious corona discharge in water and apparatus for making the same. CZ9601596. Czech Republic. Patent document. 1997-08-13.

14. Šunka P, Babický V. Generation method of focused shock wave by electric discharge in a liquid and apparatus for making the same. CZ20001513. Czech Republic. Patent document. 2002-08-14.

15. Beneš J, Šunka P, Králová J, Kašpar J, Poučková P. Biological Effects of Two Successive Shock Waves Focused on liver tissues and melanoma cells. Physiological research. 2007, Vol. 56, pp. S1-S4. Available at WWW: <http://www.biomed.cas.cz/physiolres $>$.

Received May 17, 2011. Accepted February 20, 2012. 